\title{
Network abnormalities in generalized anxiety pervade beyond the amygdala-pre-frontal cortex circuit: Insights from graph theory
}

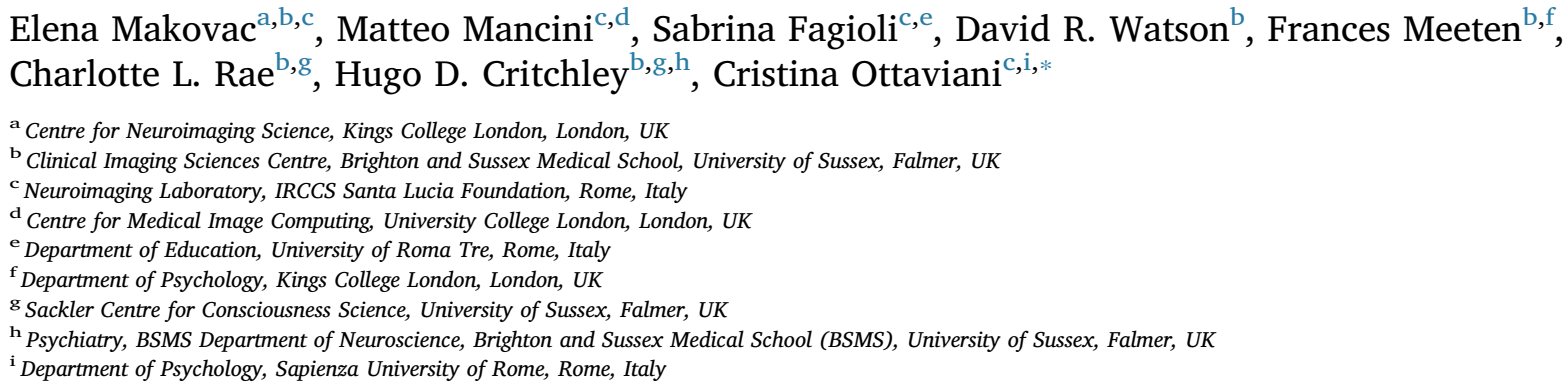

\section{A R T I C L E I N F O}

\section{Keywords:}

Generalized anxiety disorder

Functional connectivity

Graph theory

Longitudinal

Global efficiency

Network-based statistics

\begin{abstract}
A B S T R A C T
Generalized anxiety disorder (GAD) has excessive anxiety and uncontrollable worry as core symptoms. Abnormal cerebral functioning underpins the expression and perhaps pathogenesis of GAD:. Studies implicate impaired communication between the amygdala and the pre-frontal cortex (PFC). Our aim was to longitudinally investigate whether such network abnormalities are spatially restricted to this circuit or if the integrity of functional brain networks is globally disrupted in GAD. We acquired resting-state functional magnetic resonance imaging data from $16 \mathrm{GAD}$ patients and 16 matched controls at baseline and after 1 year. Using network modeling and graph-theory, whole-brain connectivity was characterized from local and global perspectives. Overall lower global efficiency, indicating sub-optimal brain-wide organization and integration, was present in patients with GAD compared to controls. The amygdala and midline cortices showed higher betweenness centrality, reflecting functional dominance of these brain structures. Third, lower betweenness centrality and lower degree emerged for PFC, suggesting weakened inhibitory control. Overall, network organization showed impairments consistent with neurobiological models of GAD (involving amygdala, PFC, and cingulate cortex) and further pointed to an involvement of temporal regions. Such impairments tended to progress over time and predict anxiety symptoms. A graph-analytic approach represents a powerful approach to deepen our understanding of GAD.
\end{abstract}

\section{Introduction}

Generalized anxiety disorder (GAD) is a chronic condition characterized by excessive anxiety, in which uncontrollable anticipation of negative outcomes (i.e. worry) may develop as a response to manage emotional distress. GAD is the most frequent anxiety disorder in primary care, imposing an enormous human and economic burden on society (Hoffman et al., 2008). Abnormal cerebral functioning is evident and implicated in the pathogenesis of anxiety, with a clear role of the amygdala (Mochcovitch et al., 2014). Indeed, functional brain imaging studies show heightened activation of the amygdala across anxiety disorders when compared to healthy controls (HC). Similarly, enhanced amygdala reactivity correlates with trait anxiety in both clinical and healthy populations. Thus, hyper-responsiveness of the amygdala is putatively a trans-diagnostic neural correlate of dispositional anxiety (e.g. Etkin et al., 2009). The role of the amygdala in the pathophysiology of GAD is less clear, with some studies reporting overreactivity (e.g. greater anticipatory amygdala activity preceding aversive and neutral stimuli; Nitschke et al., 2009), and others diminished activity of the amygdala, for example during the evaluation of angry faces (Blair et al., 2008). Similarly, other studies have failed to report a hyperactivation of the amygdala during the presentation of threatening stimuli in GAD (Monk et al., 2006; Palm et al., 2011). The results appear to be more coherent in pediatric GAD, where hyperactivation of

\footnotetext{
* Corresponding author at: Department of Psychology, Sapienza University of Rome, Via dei Marsi, 78 , 00185 Rome, Italy.

E-mail address: cristina.ottaviani@uniroma1.it (C. Ottaviani).
} 
the amygdala is evident during the elaboration of emotional stimuli and correlated with the severity of GAD symptoms (Monk et al., 2008; McClure et al., 2007).

On its own, the quantification of amygdala dysfunction yields limited insights to the pathophysiology of anxiety disorders in general and of GAD in particular (Paulus and Stein, 2006). In recent years, understanding of GAD pathophysiology has been enriched by the investigation of abnormal patterns of communication within and between brain networks, capitalizing upon resting state functional connectivity approaches (Sylvester et al., 2012). Moreover, resting-state connectivity tools can be successfully used to demonstrate functional differences and similarities in neural characteristics of distinct anxiety disorders (Peterson et al., 2014). Aberrant communication between amygdala and pre-frontal cortex (PFC) emerges repeatedly as a signature of GAD (Makovac et al., 2016a; Mochcovitch et al., 2014). Crucially, in nonclinical populations, amygdala activity is tonically suppressed by inhibitory inputs from the PFC, enabling the efficient regulation of emotional states (Nomura et al., 2004). Therefore, the emotional dysregulation typical of GAD may plausibly reflect dysfunctional communication between PFC and amygdala, in which the failure of the PFC to down-regulate the amygdala in safe contexts leads to the maintenance of core symptoms of worry and anxiety (Etkin et al., 2009; Makovac et al., 2016a). Such a mechanism illustrates how specific patterns of network dysfunction can contribute to core deficits in cognitive and affective functioning that underlie the expression of clinical symptoms.

Nevertheless, focusing only on the communication between PFC and amygdala (as with focusing on amygdala activation alone) may be too reductive and obscure the recognition of more subtle abnormalities distributed across the brain, of potentially equivalent pathoaetiological significance. Indeed, GAD involves dysfunction of cognitive and emotion regulation processes relying on distributed brain regions spanning multiple lobes (Menon, 2011). For example, other studies have reported a crucial role of the communication between amygdala and temporal pole in GAD (Li et al., 2016). Similarly, recent data have pointed to an involvement of the communication between amygdala and temporal areas in the mediation of the negative affectivity that accompanies worry in GAD (Makovac et al., 2018).

A graph theory analytic approach permits a more global perspective on functional neural connectivity, as only large-scale brain network analytics can provide integrative models of cognitive and affective dysfunction in GAD (Menon, 2011). Within this network-modeling framework, brain regions are represented as nodes of a mathematical graph, and the functional couplings between them constitute its edges (Bullmore and Sporns, 2009; Rubinov and Sporns, 2010). Metrics from graph theory are employed to characterize specific network properties including segregation, i.e. the capability of specialized local processing, and integration, i.e. the capability of distributed global processing. Importantly, a consequence of network organization is that it supports spreading processes between connected regions. It follows that a localized brain dysfunction can cause pathological alterations within regions that are distant, yet functionally linked to the original site of dysfunction (Fornito et al., 2015).

Human 'neural connectomics' has yielded plausible biomarkers for Alzheimer's disease (Bergeron et al., 2016) and psychiatric disorders including schizophrenia (Kambeitz et al., 2016), social anxiety disorder (Yun et al., 2017), post-traumatic stress disorder (Lei et al., 2015), and major depression (Gong and He, 2015). Despite the promise of this approach, and the conceptualization of anxiety disorders as "dysfunction in brain networks" (Sylvester et al., 2012), to date no study has yet applied graph theory to whole brain network connectivity in GAD patients. The present paper addresses this need. We examined whole brain functional connectivity in GAD patients and HC by applying specific quantitative graph measures. We hypothesized that global and local brain network topological properties are disrupted in GAD compared to controls, and that these disruptions extend beyond the PFC-amygdala interactions proposed as a canonical circuit dysfunction. Given the absence of previous studies applying this approach in GAD, we opted for both a data- and theory-driven approach. The latter specifically involved the exploration of brain regions that have emerged as playing a significant role in prior studies on the neurobiology of GAD, i.e., regions within the PFC, and cingulate gyrus (e.g., Makovac et al., 2016a; Via et al., 2018).

The progression of a clinical anxiety disorder is directly coupled to time dependent expression and modification of symptoms (van Beljouw et al., 2010). Correspondingly, we tested for changes in organizational features of whole brain networks at two time points over a 1-year period. Abnormalities in global network organization have the capacity to be clinically important biomarkers for disease progression, for example mapping the transition to psychosis in an at-risk sample (Lord et al., 2012) or mirroring daily affective instability in remitted patients with major depressive disorder (Servaas et al., 2017). In a previous study, we found that longitudinal changes in dorsolateral PFCamygdala functional connectivity mirrored changes in anxiety symptoms in GAD patients over time (Makovac et al., 2016b). Here, we aimed to extend these findings moving "from connectivity to connectomics".

\section{Materials and methods}

\subsection{Participants}

The present study is based on a secondary analysis of data from a larger longitudinal fMRI study (Makovac et al., 2016b). The study was approved by the National Research Ethics Service for the UK National Health Service with university sponsorship granted via the Brighton and Sussex Medical School Research Governance and Ethics Committee. All participants provided written informed consent at both time points. The final sample undergoing both assessments encompassed 16 patients (14 women; mean age $=29.6 \pm 7.5$ years) who met DSM-IV diagnostic criteria for GAD and $16 \mathrm{HC}$ (13 women; mean age $=28.1 \pm$ 10.1 years). The average illness duration at time 0 was $16.8 \pm 8.0$ years. Patients and controls were medication free, with the exception of two patients with GAD who used long-term medications (one citalopram, one pregabalin) at both sessions of the study. Wash-out was not applied. At time 0, forty individuals (19 GAD, $21 \mathrm{HC}$ ) were recruited by public advertisement; after one-year (time 1), eight participants had dropped-out from the study ( $3 \mathrm{GAD}$ and $5 \mathrm{HC}$ ). All participants were right-handed and native English speakers. Exclusion criteria were: age younger than 18 years, past head injury or neurological disorders, prior history of major medical or psychiatric disorder (other than GAD for the patient group), cognitive impairment, history of substance or alcohol abuse or dependence, diagnosis of heart disease, obesity, pregnancy, claustrophobia or other general magnetic resonance imaging (MRI) exclusions. None of our participants had a formal diagnosis of comorbid major depressive disorder.

\subsection{Procedure}

At both time 0 and time 1, all participants underwent the Structured Clinical Interview for DSM-IV to confirm or exclude a current diagnosis of GAD. Then, participants completed a series of online questionnaires and underwent the MRI protocol. Participants completed the same procedure about 1 year later (time 1) (average time between sessions $=10.5 \pm 2.2$ months). The 1-year time frame was chosen for both practical and theoretical reasons. Given our small sample size and difficulties in recruiting anxious patients for a brain imaging study, we opted for a time frame that allowed us to detect changes in symptoms (DSM-5 criteria require a minimum of 6 months of persistent worry for the diagnosis of GAD), while at the same time minimizing the risk of losing patients at follow-up. 


\subsection{Questionnaires}

A series of lifestyle (nicotine consumption, alcohol and caffeine intake, and physical activity) socio-demographic (age, years of education), and dispositional questionnaires (STAI, PSWQ) were administered at both time points (Makovac et al., 2016a).

The State Trait Anxiety Inventory (STAI; Spielberger, 1983) consists of two 20-item self-report measures to assess state ("I am tense") and trait ("I feel like a failure") levels of anxiety. Respondents indicate how they feel right now (state version) or how they generally feel (trait version) using four-point Likert scales.

The Penn State Worry Questionnaire, (PSWQ; Meyer et al., 1990), is a 16-item self-report questionnaire mainly focused on future outcomes ("As soon as I finish one task, I start to worry about everything else I have to do") and commonly used to assess the dispositional tendency to engage in worrisome thoughts.

\section{4. fMRI design}

At both time points, participants underwent a 5 min resting-state period, during which they were instructed to rest with their eyes open without thinking of anything in particular and not falling asleep. Previous studies have shown that the resting condition (i.e. eyes open vs eyes closed) can affect resting state fMRI reliability (e.g. Patriat et al., 2013). With the aim of replicability, we have opted for the most commonly used condition in this specific field of research i.e., eyes open. At both time points, the resting-state periods were followed by a series of tasks beyond focus of the present article and described elsewhere (Ottaviani et al., 2016).

\subsection{MRI acquisition and pre-processing}

MRI images were acquired on a 1.5-Tesla Siemens Magnetom Avanto scanner (Siemens AG, Munich, Germany). Functional datasets used $\mathrm{T} 2 \times$ weighted echoplanar imaging (EPI) sensitive to blood oxygenation level dependent (BOLD) signal (TR $=2.52 \mathrm{~s}$, TE $=43 \mathrm{~ms}$, flipangle $90^{\circ}$, 34 slices, $3 \mathrm{~mm}$ slice thickness, $192 \mathrm{~mm}$ FOV, voxel size $3 \times 3 \times 3 \mathrm{~mm}$ ). T1 weighted (MPRAGE) volumes $(0.9 \mathrm{~mm}$ isometric voxels, 192 sagittal slices, repetition time $11.4 \mathrm{~ms}$, echo time $4.4 \mathrm{~ms}$, inversion time $300 \mathrm{~ms}$ ) were also acquired from each participant and visually reviewed to exclude the presence of macroscopic artefacts. T1 data were pre-processed using FreeSurfer (v.5.3.0, https://surfer.nmr. mgh.harvard.edu) for tissue classification and whole-brain parcellation using the Desikan-Killiany atlas into 82 (14 subcortical, 68 cortical) anatomical regions (Desikan et al., 2006). Resting-state data were preprocessed using FSL (v.5.0.7, https://fsl.fmrib.ox.ac.uk/fsl/). Briefly, the first four volumes were discarded to ensure field homogeneity, while the remaining volumes were corrected for ascendant slice-timing, entered to MCFLIRT to estimate motion parameters, and spatially smoothed using a Gaussian kernel with a width of $5 \mathrm{~mm}$. AROMA was used to reject motion-related artefacts using an independent component approach based on MELODIC (Pruim et al., 2015). In line with recent papers (Alakörkkö et al., 2017; Chen and Calhoun, 2018; Liu et al., 2017), we limited the width of the spatial smoothing to reach a tradeoff between avoiding overestimation and enhancing motion artefacts detection (Pruim et al., 2015). CSF and white matter signals were regressed out. Lastly, the clean data were co-registered using an inverse transformation from the EPI to the native space in order to match the parcellation scheme.

\subsection{Network modeling}

The resulting pre-processed images were used for constructing the connectivity matrices. For each region, the average BOLD time-course was calculated using the maximum-overlap discrete wavelet transform (MODWT) such that a time-course was decomposed into specific frequency bands. Following previously published works (Bassett and Bullmore, 2006), we selected the coefficients of the second scale, which provided information on the frequency band $0.05-0.1 \mathrm{~Hz}$. Then, for every possible pair of regions, the correlation between the wavelet coefficients and the related $p$-value were calculated and arranged in matrices. We then used false discovery rate (FDR) with $p<0.05$ on the obtained $p$-values to take into account multiple comparison and we built a connectivity matrix using only the correlation values that passed the FDR test (Bassett et al., 2011b). We applied this statistical threshold to all the matrix elements regardless of the sign of the correlation. In this way, we were able to describe brain networks by means of mathematical graphs, where each node represented a region from the Desikan-Killiany atlas and each edge the presence or absence of functional coupling.

\subsection{Network-based statistics}

To characterize differences edge-wise while taking into account multiple comparisons, we used a network-based statistics (NBS) approach for computing a mixed two-way ANOVA (Zalesky et al., 2010). Briefly, for every edge in the graph, the $F$-test was computed edge-wise with the appropriate design matrix. The resulting pseudo-adjacency matrix was binarized using an arbitrary threshold $(F>5)$ on the statistics (Smiths and Nichols, 2009), and the size of the largest connected component was calculated (here the size of a connected component is the number of its vertices). Using a permutation approach $(10,000$ permutations), the matrices were randomly reassigned, and the statistics were iteratively re-computed as well as the size of the largest component. As a result, a $p$-value was obtained as the ratio between the number of random components larger than the real one and the total number of permutations computed. Using NBS results, we were able to define a sub-network (i.e. a subset of regions with the relative connections) showing significant differences between the considered groups and time-points.

\subsection{Graph measures}

We characterized the obtained brain networks from the global and local perspectives using measures inherited from graph theory (Bullmore and Sporns, 2009). All the mentioned measures were computed using MATLAB and Brain Connectivity Toolbox (v.2017-15-01, https://sites.google.com/site/bctnet/).

As global measures, we calculated the average clustering coefficient, which quantifies how dense the connections are among neighbor nodes; the global efficiency, which is inversely related to the characteristic path length (i.e. the average length of all the shortest paths in a graph); and the overall functional connectivity (calculated as the average of all the non-zero elements). To take into account effects driven by differences in the number of connections, the density was also computed (van den Heuvel et al., 2017).

As local measures, we calculated for each node the degree, which is the number of connections; the betweenness centrality, which measures how much the node is involved in efficient paths; and the local efficiency, which is the inverse of the length of the shortest paths passing through a given node (Rubinov and Sporns, 2010).

\subsection{Statistical analysis}

Data analysis was performed with SPSS 22.0 for Windows (SPSS Inc, USA). First, a series of $t / \chi^{2}$ tests were performed to look at baseline differences in potential confounding variables (e.g., BMI, years of education) between patients with GAD and HC who completed the protocol at both time points (Makovac et al., 2016b).

Second, effects of Group, Time, and Group $\times$ Time interaction on clinical scores (STAI, PSWQ) were examined by analyses of variance (ANOVA). 

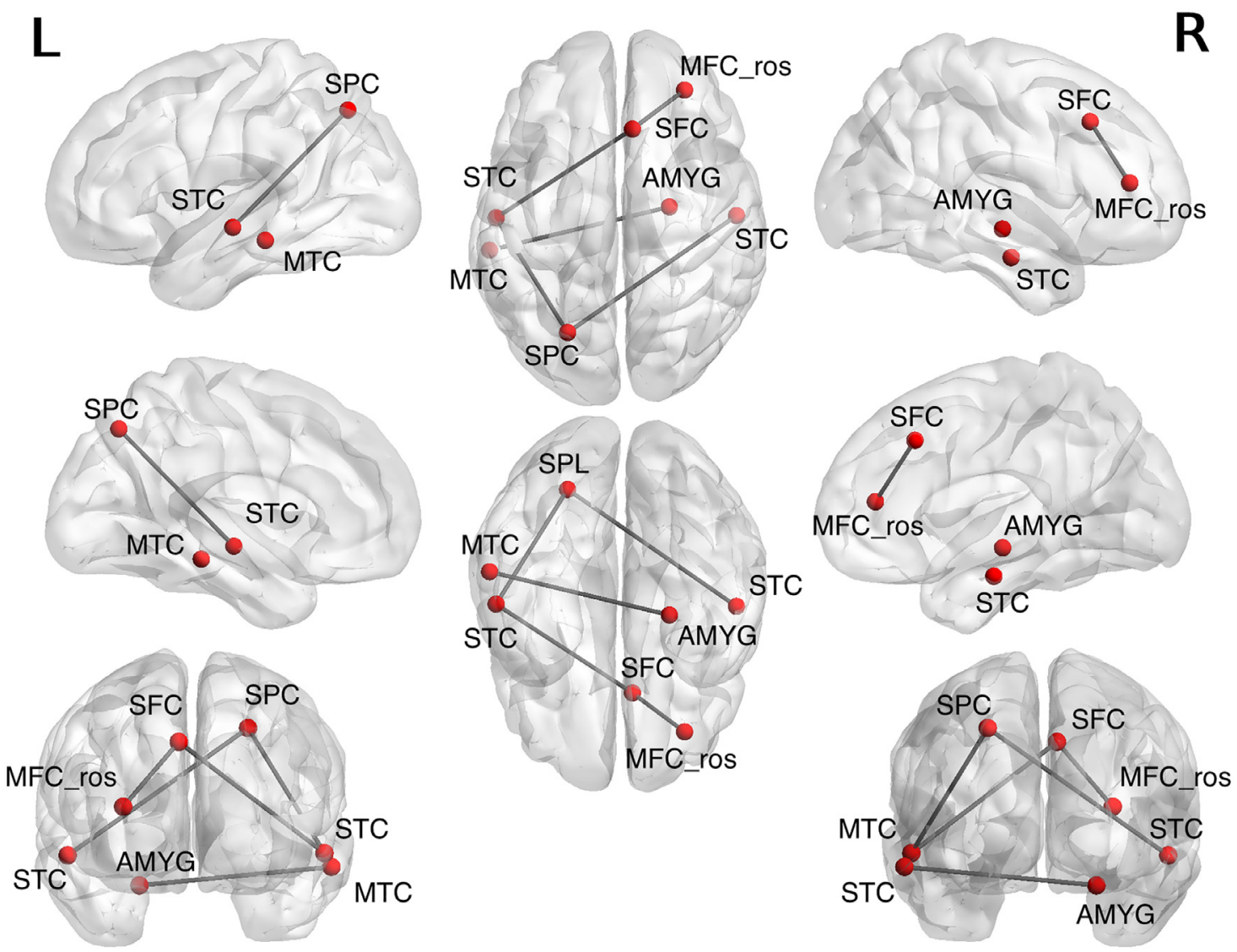

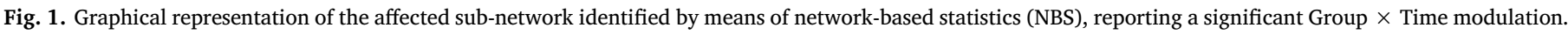

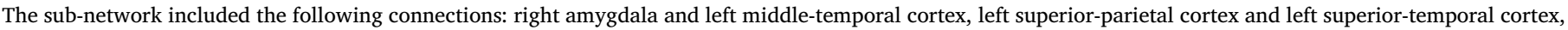

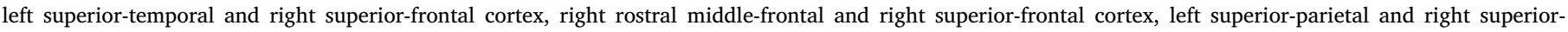

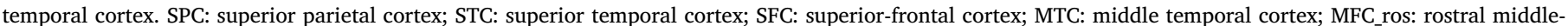
frontal cortex; AMYG: amygdala.

Third, effects of Group, Time, and Group $\times$ Time interaction on region-to-region connectivity were identified using NBS. Given that NBS does not reveal the direction of effects, significant results were further explored using graph theory measures. Specifically, we compared properties of the brain networks of patients with GAD and HC by overall mixed multivariate analyses of variance (MANOVA) separately for global and local measures to minimize the likelihood of type-I errors. The analyses were performed on a pool of regions of interest (ROI) selected on the basis of: a) existing theoretical and empirical knowledge (amygdala, PFC, posterior cingulate (PCC), rostral-anterior cingulate (ACC), and caudal-ACC of both hemispheres); and b) results of the NBS. Due to low statistical power, which reduces the chance to detect any true effect (Button et al., 2013), the univariate tests following the MANOVA analyses were not corrected for multiple comparisons.

In these analyses, the global (i.e., average clustering coefficient, global efficiency, and overall functional connectivity) or local (i.e., betweenness centrality, local efficiency, and degree) measures for each ROI were used as dependent variables. Group (GAD, HC) was used as between-subjects factor, and the time of assessment (time 0 , time 1) was used as withinsubjects factor. Preliminary checks were conducted to ensure that there was no violation of the assumptions of normality, linearity, and homogeneity of variances of the considered variables. Each individual overall MANOVA was followed by a series of post-hoc ANOVAs to measure differences between groups (i.e., main effect) and selective modulation within patterns of connectivity by the time of assessment among groups (i.e., interaction term). The level of statistical significance used for these analyses was defined as $p<0.05$.

Lastly, we explored the relationship between the global and local properties of the obtained brain network and the continuous measure of dispositional worry and anxiety in patients with GAD. Toward this aim, partial correlations (adjusting for age) were used to examine the association between scores on the PSWQ and STAI and the measures of global and local connectivity that emerged as different between patients with GAD and HC at time 0 and time 1, separately. Given the high number of correlations, only those that survived Bonferroni correction are reported. Then, in order to verify the magnitude of the correlations over time, $t$-tests for dependent correlations were performed (Steiger, 1980).

\section{Results}

\subsection{Group differences}

The groups did not differ in age, years of education, sex distribution, nicotine consumption, alcohol and caffeine intake, physical activity, or body-mass index (see Makovac et al., 2016b for demographics and clinical scores at time 0 and time 1). During the 1-year interscan gap, 1 patient with GAD started yoga-mindfulness and 2 of them started cognitive-behavioral therapy (CBT). Overall, results changed neither after exclusion of the two medicated patients, nor when the three patients who had begun CBT or yoga-mindfulness were excluded from the analyses, with one graph theory parameter exception for the latter (see Section 3.6). 


\subsection{Effects of Group, Time, and Group $\times$ Time on clinical scores}

As to the state version of the STAI, main effects of Group, $F$ $(1,30)=27.30, p<0.001, \eta_{p}{ }^{2}=0.48$ and Time, $F(1,30)=4.21$, $p=0.49, \eta_{p}{ }^{2}=0.13$ were observed, where patients with GAD reported significantly higher scores compared to $\mathrm{HC}$, independently of the time point $(\mathrm{GAD}=46.92$ (9.67); $\mathrm{HC}=32.41$ (8.06); $p<0.001)$. Moreover, state anxiety was higher at time 1 (38.78 (1.48) compare to time 0 (38.78 (1.75)), irrespective of Group $(p=0.49)$. We did not observe a significant Group $\times$ Time interaction.

As to the trait version of the STAI, only a main effect of Group, $F$ $(1,30)=39.64, p<0.001, \eta_{p}{ }^{2}=0.57$ emerged, where patients with GAD had higher scores compared to $\mathrm{HC}(\mathrm{GAD}=52.58$ (1.91); HC $=35.59$ (1.91); $p<0.001)$.

The model with PSWQ as the outcome yielded only a significant effect of Group, $F(1,28)=46.74, p<0.001, \eta_{p}{ }^{2}=0.97$, with GAD patients reporting higher scores compared to $\mathrm{HC}(\mathrm{GAD}=66.21$ (2.75); $\mathrm{HC}=40.50$ (2.57); $p<0.001)$.

\subsection{Network-based statistics}

No main effects of Group or Time emerged for region-to-region connectivity. NBS showed a significant Group $\times$ Time interaction in a sub-network that included the following connections: right amygdala and left middle-temporal cortex; left superior-parietal cortex and left superior-temporal cortex; left superior-temporal and right superiorfrontal cortex; right rostral middle-frontal and right superior-frontal cortex, left superior-parietal and right superior-temporal cortex (Fig. 1). Post-hoc univariate analysis on these connections did not highlight significant effects. However, we proceeded by exploring the global and local perspectives of the sub-network obtained with the NBS approach (Section 2.8).

\subsection{Effects of Group, Time, and Group $\times$ Time on global and local measures of connectivity}

Significant findings deriving from global and local measures of connectivity are summarized in Table 1.

\subsection{Global measures of connectivity}

A main effect of Group, approaching statistical significance, was evident for global efficiency, $F(91,30)=4.09, p=0.052, \eta_{p}{ }^{2}=0.12$ ), with GAD showing lower global efficiency compared to HC $(0.35 \pm 0.18$ and $0.37 \pm 0.19$, respectively). No significant Time $\times$

Table 1

Significant results for graph theory measures.

\begin{tabular}{|c|c|c|c|}
\hline & T0-T1 & T0 & $\mathrm{T} 1$ \\
\hline \multicolumn{4}{|l|}{ Global measures } \\
\hline Global efficiency & & $\mathrm{GAD} \downarrow$ & GAD $\downarrow$ \\
\hline \multicolumn{4}{|l|}{ Local measures } \\
\hline \multicolumn{4}{|l|}{ Betweenness centrality } \\
\hline $\mathrm{R}$ amygdala & $\mathrm{GAD} \uparrow$ & & \\
\hline L middle-temporal cortex & GAD $\downarrow$ & & $\mathrm{GAD} \downarrow$ \\
\hline L lateral orbito-frontal cortex & $\mathrm{HC} \downarrow$ & $\mathrm{GAD} \downarrow$ & \\
\hline L posterior cingulate cortex & $\mathrm{GAD} \uparrow$ & $\mathrm{GAD} \downarrow$ & GAD 1 \\
\hline $\mathrm{L}$ rostral anterior cingulate cortex & & $\mathrm{GAD} \uparrow$ & \\
\hline \multicolumn{4}{|l|}{ Degree } \\
\hline R superior-frontal cortex & & $\mathrm{GAD} \downarrow$ & $\mathrm{GAD} \downarrow$ \\
\hline L superior-parietal cortex & & $\mathrm{GAD} \downarrow$ & GAD \\
\hline L middle-temporal cortex & & $\mathrm{GAD} \downarrow$ & $\mathrm{GAD} \downarrow$ \\
\hline
\end{tabular}

Note. $\mathrm{L}=$ left; $\mathrm{R}=$ right; $\mathrm{GAD}=$ generalized anxiety disorder; $\mathrm{HC}=$ healthy controls; T0-T1 = within-group differences between time 0 and time 1 ; $\mathrm{T} 0=$ between-group differences at time $0 ; \mathrm{T} 1=$ between-group differences at time $1 ; \uparrow=$ increase; $\downarrow=$ decrease.
Group interactions were evident for any of the considered global measures. No main effects of Group or Time emerged for average clustering coefficient and overall functional connectivity.

\subsection{Local measures of connectivity}

Significant Group $\times$ Time interactions were identified for local measures of connectivity (Fig. 2). When testing the same regions obtained from the NBS, our MANOVA revealed significant Group $\times$ Time interactions for betweenness centrality measures (Wilki's $\lambda=0.63$, $\left.F=3.00, d f=5, p=0.028, \eta_{p}{ }^{2}=0.37\right)$. A series of follow-up 2-way ANOVAs (Table 1 ) revealed a significant Group $\times$ Time interaction for betweenness centrality in:

- Right amygdala $\left(F(1,30)=5.63, p=0.024, \eta_{p}{ }^{2}=0.16\right)$, driven by an increase in betweenness centrality from time $0(82.50 \pm 51.66)$ to time $1(123.62 \pm 89.02)$ in $\mathrm{GAD}, t(15)=2.45, p=0.027$, $d=0.56$.

- Left middle-temporal cortex $(F(1,30)=5.42, \quad p=0.027$, $\left.\eta_{p}{ }^{2}=0.15\right)$, driven by a decrease in betweenness centrality from time $0(150.89 \pm 56.89)$ to time $1(108.43 \pm 55.24)$ in GAD, $t$ $(15)=2.38, p=0.031, d=0.75$, and by a significant difference between HC $(183.63 \pm 81.03)$ and GAD at time $1, t(15)=3.33$, $p=0.005, d=1.08$.

When testing a priori (theory-driven) regions of the PFC and cingulate gyrus, this MANOVA revealed an overall Group $\times$ Time interaction for betweenness centrality, Wilki's $\lambda=0.26, F(1,13)=3.89$, $p=0.004, \eta_{p}{ }^{2}=0.74$. Follow-up ANOVAs (Table 1) revealed a Group $\times$ Time interaction in the following areas:

- Left lateral orbito-frontal cortex $(F(1,30)=6.67, p=0.015$, $\left.\eta_{p}{ }^{2}=0.18\right)$ driven by a decrease in betweenness centrality from time $0(180.79 \pm 108.89)$ to time $1(98.93 \pm 75.24)$ in $\mathrm{HC}, t$ (15) $=2.60, p=0.02, d=0.87$ and a significant difference between GAD and HC at time 0 , where GAD showed lower betweenness centrality $(106.77 \pm 64.19)$ compared to HC, $t(15)=2.46$, $p=0.03, d=0.82$.

- Left PCC, $\left(F(1,30)=8.72, p=0.006, \eta_{p}{ }^{2}=0.23\right)$, driven by a significant difference in betweenness centrality between GAD and HC at time 0 (GAD: $59.13 \pm 42.87$ vs HC: $107.86 \pm 86.36), \quad t$ $(15)=2.11, p=0.052, d=0.71)$, a significant increase in $b e$ tweenness centrality in GAD from time 0 to time 1 (GAD time $1=139.17 \pm 75.77, t(15)=3.22, p=0.006, d=1.30)$, and a significant difference between patients with GAD and HC at time 1 (HC time $1=95.03 \pm 43.82, t(15)=2.10, p=0.053, d=0.71$ ), where patients presented lower betweenness centrality at time 0 and higher betweenness centrality at time 1 .

- Left rostral ACC $\left(F(1,30)=4.61, p=0.040, \eta_{p}{ }^{2}=0.13\right)$, driven by a significant difference between GAD (158.83 \pm 97.33) and HC $(102.69 \pm 46.67)$ at time $0, t(15)=2.14, p=0.049, d=0.73)$, whereas no difference was observed at time 1 (GAD: $121.38 \pm 72.72$ vs HC: $155.25 \pm 99.78, t(15)=1.12, p=0.28$, $d=0.38)$. This difference was no longer significant $(p=0.07)$ when the three patients who had begun CBT or yoga-mindfulness between time 0 and time 1 were removed from the analyses.

The MANOVA on the NBS regions for the degree measure revealed a main effect of the factor Group (Wilki's $\lambda=0.56, F=2.74$, $d f=24$, $\left.p=0.031, \eta_{p}{ }^{2}=0.44\right)$, driven by lower degree in GAD (25.43 \pm 7.09$)$ compared to HC $(31.56 \pm 9.79)$ within the right superior-frontal cortex, $\left.F(1,30)=6.50, p=0.016, \eta_{p}{ }^{2}=0.18\right)$, left superior-parietal cortex (GAD: $27.97 \pm 10.67$ vs HC: $34.06 \pm 9.70, F(1,30)=4.80$, $p=0.036, \eta_{p}{ }^{2}=0.14$ ) and left middle-temporal cortex (GAD: $26.86 \pm 10.44$ vs HC: $33.19 \pm 9.72 ; \quad F(1,30)=4.36, \quad p=0.045$, $\left.\eta_{p}{ }^{2}=0.13\right)$. 

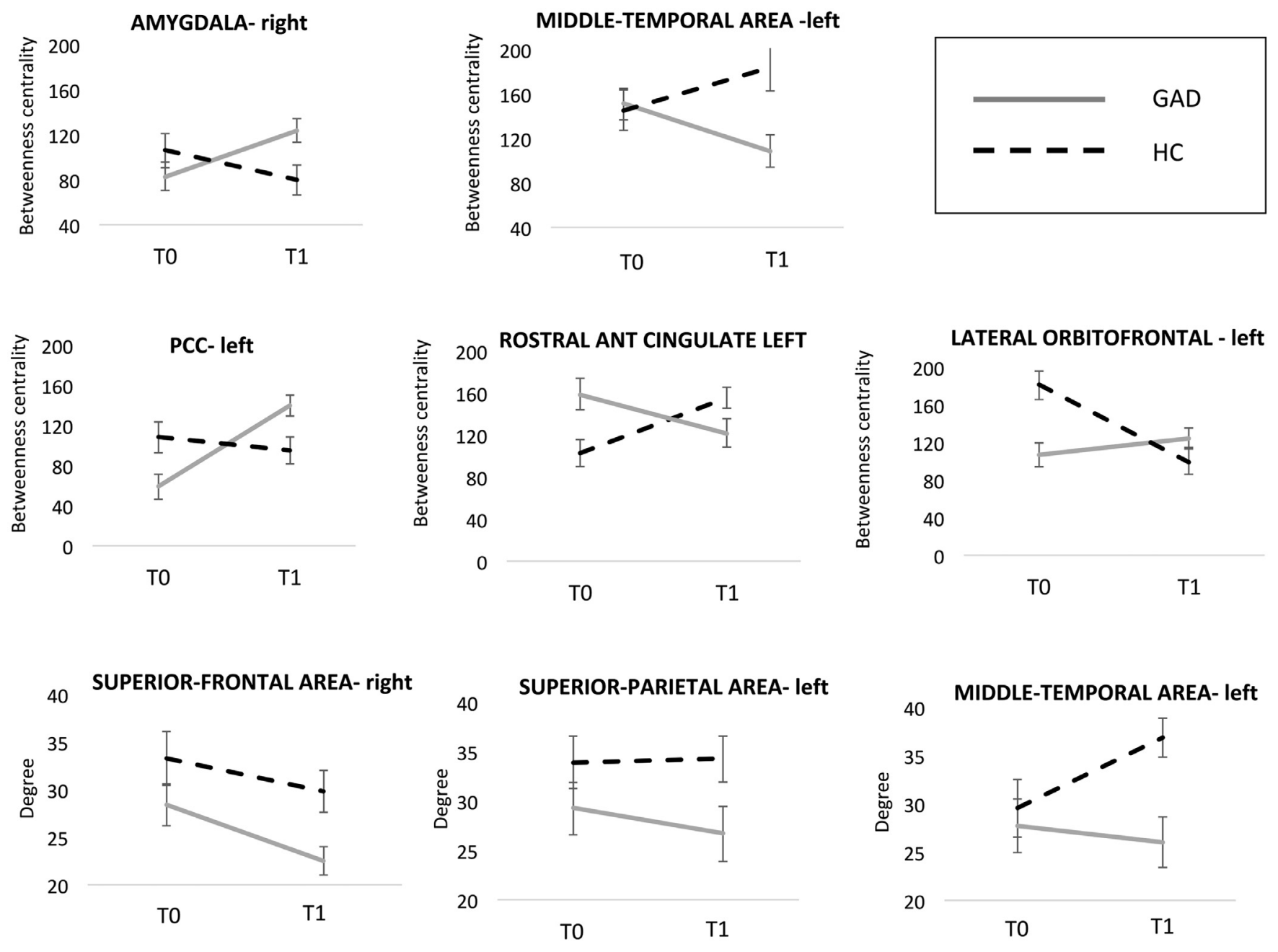

Fig. 2. Effects of Group, Time, and Group $\times$ Time interaction on graph-theory local measures.

3.6. Pattern of variation between symptoms of worry and trait anxiety and global and local measures of connectivity over time

At time 0 , trait anxiety was negatively correlated with the measure of degree $(r=-0.79, p<0.001)$ within the left superior parietal lobe. Higher dispositional worry was associated with higher betweenness centrality of the right amygdala $(r=0.62, p<0.001)$ and reduced local efficiency in the rostral ACC bilaterally ( $r s=-0.55, p s=0.002)$. No other significant associations emerged for global and local measures of functional connectivity at time 0 .

At time 1, increasing scores on both trait anxiety and dispositional worry were associated with reduced global efficiency $(r=-0.59$, $p=0.002$ and $r=-0.54, p=0.002$, respectively) and poorer local efficiency within the right ACC $(r=-0.69, p<0.001$ and $r=-0.66$, $p<0.001$, respectively).

\section{Discussion}

The present study investigated global and local properties of functional connectivity in patients with GAD and controls at two time points separated by approximately 1 year. We found evidence for both disrupted global, and local, network function in people with GAD. These disruptions remained or even increased in severity over time, and within key cortical midline structures, local dysfunction predicted anxiety symptoms. While in recent years whole brain functional connectivity has been extensively examined in social anxiety disorder (e.g., Xing et al., 2016; Yang et al., 2017; Zhu et al., 2017), this is the first study to our knowledge that applied NBS and graph theory to address the question of whether network abnormalities are spatially restricted to the PFC-amygdala circuit, or if the integrity of brain function is globally disrupted in adults with GAD. An exception is represented by one recent study that tested whether parameters from dynamic functional connectivity could reliably classify adolescents with GAD from healthy controls in the absence of other clinical measures (Yao et al., 2017). The results supported the potential of dynamic functional connectivity measures in DMN and particularly in the medial PFC as a biomarker for adolescents with GAD.

Using data-driven NBS, we characterized altered collective patterns of brain function in terms of sub-networks (Zalesky et al., 2010). This approach highlighted an extended sub-network comprising pairs of regions whose functional coupling presented a Group-by-Time modulation, encompassing right amygdala alongside temporal, parietal, and frontal areas. Next, we applied graph analysis to regions-of-interest to quantify these interactions and estimate indicators of network efficiency in GAD and controls.

Extending observations reported for other psychopathological conditions, e.g. bipolar disorders (Spielberg et al., 2016), major depressive disorder (Luo et al., 2015), addiction (Wang et al., 2015), and schizophrenia (Zhu et al., 2016), we observed overall lower global efficiency in patients with GAD compared to an age- and gender-matched HC group. Moreover, a reduction in global efficiency at time 1 was associated with higher levels of trait anxiety and dispositional worry. Global efficiency is an index of parallel information transfer averaged between all pairs of nodes in the network. Global efficiency therefore represents a general 
measure of integration and distributed processing capacity. Lower global efficiency thus suggests that the patterns of functional connectivity represent suboptimal organization and the most efficient pathways are disrupted. In our view, this is the most intriguing result of our study since decreased communication efficiency betrays a loss of normal inter-regional communications in patients with GAD, possibly underpinning impairments in cognitive control and inhibition over intrusive worrisome thoughts, linked to the attentional deficits that characterize the disorder.

Importantly, we showed that overall functional connectivity and small-world properties of the network (Watts and Strogatz, 1998), including average clustering coefficient were intact in GAD patients, reflecting intact functional segregation. Thus, decreased communication efficiency is not accounted for by overall dysconnectivity or aberrant segregation. To pursue these mechanistic insights further, we characterized the importance of determined nodes within the distributed whole brain network, i.e. drawing upon results of local measures of efficiency.

When considering local measures of efficiency, betweenness centrality emerged as a key variable expressing differences between patients with GAD and controls. Betweenness centrality captures the influence that one node holds over the flow of information between all other nodes in the network. Therefore, the higher betweenness centrality in the right amygdala, rostral-ACC, and PCC observed in GAD compared to controls likely reflects the dominance of these structures in information transfer across the network (Sporns et al., 2007). We note that a number of differences emerged in betweenness centrality in a number of regions at time 0 and time 1, mostly in patients with GAD. Given the preliminary nature of the present study, it is difficult to establish whether these differences truly reflect the fact that GAD patients are more vulnerable to volatility of core nodes and efficient paths, or whether this is only due to the lack of stability of the index of betweenness centrality compared to the other graph-theory measures we examined (Segarra and Ribeiro, 2016).

Taken together, our results reinforce the evidence for the central role played by interactions of the amygdala with cortical midline structures (ACC and PCC) in patients with GAD, who seemingly overrecruit these regions (Sylvester et al., 2012). Amygdala hyperactivity in response to emotional stimuli occurs in children as well as in adults with GAD, and positively correlates with symptom severity, and predicts symptom change (Etkin et al., 2010). Similarly, one non-clinical study using a graph theoretical approach reported positive correlations between neuroticism and the betweenness centrality of bilateral amygdala (Gao et al., 2013). Cingulate cortex and adjacent PCC are implicated in the expression of the cognitive disturbances that are often observed in GAD (e.g. attentional difficulties) (Yang et al., 2015). The present study found an inverse association between dispositional worry and local efficiency in the rostral ACC bilaterally at time 0 and between both trait anxiety and dispositional worry and local efficiency within the right ACC at time 1. The ACC is implicated in another key symptom of GAD: Autonomic dysregulation (Critchley et al., 2003; Makovac et al., 2016a). Interestingly, patients with major depressive disorder also show increased betweenness centrality in ACC and PCC. This adds to the evidence implicating these midline structures in self-processing, which is putatively the pathopsychological basis of rumination in major depression (Luo et al., 2015). Both GAD and major depression share the psychiatric expression of perseverative cognition as a core symptom. Our current finding strengthens understanding of the functional neuroanatomical substrate for rumination and worry.

We showed that GAD patients exhibited lower betweenness centrality in left lateral orbito-frontal cortex across both time points, indicating that the influence that this structure exerts on the communication between other structures in a network (likely including the amygdala) is compromised. Importantly, the orbito-frontal cortex is involved in the progression of anxiety over time (Milad and Rauch, 2007), not least through its role in extinction learning (Gottfried and Dolan, 2004). Our present results mirror findings from functional imaging and seed-based functional connectivity studies, wherein an over-active amygdala (and cingulate) is frequently coupled with PFC region, consistent with a failure in inhibitory top-down control of systems giving rise to negative affect (Etkin et al., 2010; Makovac et al., 2016a). In our study, this interpretation was further supported by the lower degree observed in right superior-frontal cortex. Nodes with high degree have denser connections, and therefore represent dominant centers for information integration (i.e. hubs). In obsessive compulsive-disorder, local degree connectivity abnormalities are shown to converge with altered local metabolism and structural differences, indicating that this measure can reliably index established alterations in network integrity or hub topography in neuropsychiatric populations (Beucke et al., 2013).

In patients with $\mathrm{GAD}$, reduced degree was also locally significant in parietal and middle-temporal areas. Within left superior parietal lobe, measures of degree negatively correlated with trait anxiety in GAD, supporting the correspondence of this measure with clinical symptoms. The superior parietal lobe is involved in sustaining attention (Corbetta et al., 1993). Moreover, lesions to this region suggest it has a key role in sensorimotor integration, by actively maintaining an internal representation of one's own body (Wolpert et al., 1998). Speculatively, core symptoms of GAD, including difficulty in maintaining attention and altered interoceptive states could be associated with compromised connectivity and efficiency in the left superior parietal lobe.

Interestingly, the left middle-temporal cortex was also characterized by lower betweenness centrality, which showed a significant decrease over time in GAD only. Overall, this means that in GAD this structure has both weaker intra-communication and communication with other structures in the brain, replicating recent results in social anxiety disorder, where this alteration was proportional to the functional impairment severity (Yun et al., 2017). The medial temporal lobe plays a crucial role in mediating emotional processes such as sensitivity to threatening cues (Davidson, 2004). This brain area may be particularly sensitive to early experiences of deprivation and neglect, as shown for example by reduced medial temporal lobe volume in anxious youths with a history of neglect (De Bellis, 2005). Indeed, Maheu and colleagues showed that these adverse experiences have an impact on the functional integrity of the medial temporal lobe (Maheu et al., 2010). We do not have data on early experiences in the present sample; therefore we can only speculate on the possible environmental contributions to such impairments in GAD.

Present results from NBS suggest a sub-network comprising coupling of right amygdala and left middle-temporal cortex; graph theory highlights an increased influence of the amygdala (high betweenness centrality) and a decreased influence of the left middle-temporal cortex (low betweenness centrality) in the network in GAD patients only over the 1 year period. A stronger positive coupling between amygdala and middle temporal gyrus is observed in bipolar patients during the depressed phase of the illness compared to controls (Cerullo et al., 2012). Moreover, studies suggest that emotional events are better remembered compared to neutral events, in part because of the interaction between amygdala and hippocampus within medial temporal lobe (Dolcos et al., 2004). Again, it is possible that the enhanced role of the amygdala in the network that we observed in GAD is responsible for the negative emotional valence of spontaneous cognition in this population. In line with this hypothesis, higher dispositional worry was associated with higher betweenness centrality of the right amygdala in participants with GAD at time 0 .

A limitation of the present study is that the sample size was relatively small, due to difficulties in recruiting unmedicated patients with GAD. This might be a problem, especially in studies involving complex network modeling (Kolaczyk and Krivitsky, 2015). Second, our study is correlational and looks at spontaneous changes in whole-brain functional connectivity over time, thus incorporating significant withinsubject variability over time as a potential confound (Bijsterbosch et al., 
2017). More generally, test-retest reliability of fMRI results is limited. However, precautions can be taken to improve the significance of longitudinal imaging data in psychiatric research (e.g., Fournier et al., 2014). For example, we carefully avoided using global signal regression, which is known to decrease the reliability of graph measures derived from resting state data (Andellini et al., 2015). We acknowledge that the clinical relevance of our results might have been greater if these metrics were highly stable in $\mathrm{HC}$ and more variable in patients with GAD. However, Fig. 2 shows region characterized by stronger fluctuations in patients compared to HC and regions characterized by fluctuations of the same entity but in opposite directions between the two groups. In interpreting the results, it is also important to bear in mind that our measures do not allow the quantification of the directional causality of interactions among brain regions. Third, the univariate tests following the MANOVA analyses were not corrected for multiple comparisons. Although this decision was made to avoid the risk of type II errors, the lack of adjustment needs to be acknowledged as a limitation of the study. Fourth, resting-state functional brain connectivity is not static (Chang and Glover, 2010), therefore it is possible that resting state periods longer than 5 min would have better captured dynamic network topology changes. A systematic review of the literature on the test-retest reliability of resting state fMRI studies report that $5 \mathrm{~min}$ is the minimum length to obtain adequate reliability (Zuo and Xing, 2014). However, reliability and similarity can be greatly improved by increasing the scan lengths from $5 \mathrm{~min}$ up to $13 \mathrm{~min}$ (Birn et al., 2013). We opted for relatively short periods due to difficulties in keeping patients with GAD in the scanner, and to decrease the likelihood of dropouts at follow up. Replication with longer scan lengths, or using simultaneous multislice acquisitions to acquire more data in the same period of time, is warranted to fully ascertain the meaningfulness of the present results. The final limitation regards the parcellation scheme used in order to define the nodes of the structural or functional network. Unfortunately, there is no consensus at the moment on a meaningful definition of regional parcellation (Grayson et al., 2017). The two main strategies are given by either using anatomical landmarks or data-driven clustering (de Reus and van den Heuvel, 2013). In this study, we opted for the Desikan-Killiany atlas (Desikan et al., 2006), which is based on sulcal and gyral anatomy. Our choice is motivated mainly by the more intuitive neurobiological interpretation (Arslan et al., 2018) and the large adoption of this atlas in connectomic studies (van den Heuvel et al., 2017). An important additional consideration to bear in mind is the effect of spatial resolution: although it would be desirable to be able to discriminate between different parts of an anatomical structure, it has been shown that higher parcellation resolution leads to less reproducible metrics (Fornito et al., 2010; Bassett et al., 2011a; Cammoun et al., 2012), an obvious pitfall for a longitudinal study.

Limitations notwithstanding, this is the first study that evaluates alterations in GAD at global and local levels, and their evolution over time. By applying a data-driven approach the present study showed network alterations in local and whole-brain connectivity in patients with GAD. Global efficiency changes reflect that activity gets 'stuck in hubs or sub-networks' in this population. In terms of local changes, there are several specific circuits beyond amygdala-PFC that have hitherto been neglected. We identify lower degree (i.e. reduction of within-circuit communication) within specific circuits that include the middle-temporal cortex, which is involved in sensitivity to threatening cues, and within superior-parietal cortex, which supports sustained attention and aspects of internal body representation. Also in GAD, structures that are implicated in the core symptoms of autonomic dysregulation and intrusive worrisome thoughts appear to become more dominant as network nodes, at the expense of pre-frontal areas, possibly accounting for inhibitory deficits. Most of these alterations progressed over time in GAD and correlated with subjective reports of anxiety. Importantly, our results are coherent with neurobiological models of GAD and add another aspect to our understanding of the disorder. The fact that we found alterations in same areas/circuits only in our patient group at both time points highlights the clinical relevance of such regions for GAD and opens avenues for future investigations specifically aimed at targeting them. However, as is the case for most neuroimaging measures, the inference at individual level remains challenging. Following the increasing interest in multivariate-pattern analyses (MVPA) in recent years, and its potential in allowing inferences at the level of the individual rather than the group, we speculatively hypothesize that the MVPA approach could be applied to graph measures, improving its clinical applicability.

Current results also leave a series of open questions for future use of graph theory in this population: do people with GAD have altered neural structure that causes global efficiency differences? Is there a genetic contribution? Is there a contribution of developmental life experience? The present demonstration of altered global, and local, processing in GAD provides a foundation upon which graph theory can be used to gain deeper insight to these questions, in the ultimate aim to translate this knowledge into clinical practice, preventing alterations in, and restoring lost, network function. In fact, despite the recent advances in neuroimaging research, the classification of psychiatric disorders still relies on clinical interview assessments. We believe that graph theory measures (especially those extracted from specific nodes of interest) hold great potential as biomarkers of the disease and predictors of treatment outcome.

\section{Funding}

This work was supported by the Italian Ministry of Health (GR2010-2312442; GR2011-02348232).

\section{Authors' contributions}

C.O., H.D.C., E.M., and M.M. contributed to the conception and design of the study. E.M. and D.R.W. conducted the study. E.M. and M.M. carried out the imaging analysis, data interpretation, and drafted the manuscript. C.L.R. and S.F. advised on the data analysis, contributed to the interpretation of the data, and revised the manuscript for important intellectual content. All authors gave final approval of the version to be published.

\section{Conflict of interest}

The authors report no biomedical financial interests or potential conflicts of interest.

\section{Ethics approval}

The study was approved by the National Research Ethics Service for the UK National Health Service with university sponsorship granted via the Brighton and Sussex Medical School Research Governance and Ethics Committee.

\section{References}

Alakörkkö, T., Saarimäki, H., Glerean, E., Saramäki, J., Korhonen, O., 2017. Effects of spatial smoothing on functional brain networks. Eur. J. Neurosci. 46, 2471-2480.

Andellini, M., Cannatà, V., Gazzellini, S., Bernardi, B., Napolitano, A., 2015. Test-retest reliability of graph metrics of resting state MRI functional brain networks: a review. J. Neurosci. Methods 253, 183-192.

Arslan, S., Ktena, S.I., Makropoulos, A., Robinson, E.C., Rueckert, D., et al., 2018. Human brain mapping: a systematic comparison of parcellation methods for the human cerebral cortex. Neuroimage 170, 5-30.

Bassett, D.S., Bullmore, E., 2006. Small-world brain networks. Neuroscientist 12, 512-523.

Bassett, D.S., Brown, J.A., Deshpande, V., Carlson, J.M., Grafton, S.T., 2011a. Conserved and variable architecture of human white matter connectivity. Neuroimage 54, 1262-1279.

Bassett, D.S., Wymbs, N.F., Porter, M.A., Mucha, P.J., Carlson, J.M., et al., 2011b. Dynamic reconfiguration of human brain networks during learning. Proc. Natl. Acad. 
Sci. U S A 108, 7641-7646.

Bergeron, D., Bensaïdane, R., Laforce, R., 2016. Untangling Alzheimer's Disease clinicoanatomical heterogeneity through selective network vulnerability - an effort to understand a complex disease. Curr. Alzheimer Res. 13, 589-596.

Beucke, J.C., Sepulcre, J., Talukdar, T., Linnman, C., Zschenderlein, K., Endrass, T., et al., 2013. Abnormally high degree connectivity of the orbitofrontal cortex in obsessive-compulsive disorder. JAMA Psychiatry 70, 619-629.

Bijsterbosch, J., Harrison, S., Duff, E., Alfaro-Almagro, F., Woolrich, M., Smith, S., 2017 Investigations into within- and between-subject resting-state amplitude variations. Neuroimage 159, 57-69.

Birn, R.M., Molloy, E.K., Patriat, R., Parker, T., Meier, T.B., Kirk, G.R., et al., 2013. The effect of scan length on the reliability of resting-state fMRI connectivity estimates. Neuroimage 83, 550-558.

Blair, K., Shaywitz, J., Smith, B.W., Rhodes, R., Geraci, M., Jones, M., et al., 2008. Response to emotional expressions in generalized social phobia and generalized anxiety disorder: evidence for separate disorders. Am. J. Psychiatry 165, 1193-1202.

Bullmore, E., Sporns, O., 2009. Complex brain networks: graph theoretical analysis of structural and functional systems. Nat. Rev. Neurosci. 10, 186-198.

Button, K.S., Ioannidis, J.P.A., Mokrysz, C., Nosek, B.A., Flint, J., Robinson, E.S.J., et al., 2013. Power failure: why small sample size undermines the reliability of neuroscience. Nat. Rev. Neurosci. 14, 365-376.

Cammoun, L., Gigandet, X., Meskaldji, D., Thiran, J.P., Sporns, O., et al., 2012. Mapping the human connectome at multiple scales with diffusion spectrum MRI. J. Neurosci. Methods 203, 386-397.

Cerullo, M.A., Fleck, D.E., Eliassen, J.C., Smith, M.S., DelBello, M.P., Adler, C.M., et al., 2012. A longitudinal functional connectivity analysis of the amygdala in bipolar I disorder across mood states. Bipolar Disord. 14, 175-184.

Chang, C., Glover, G.H., 2010. Time-frequency dynamics of resting-state brain connectivity measured with fMRI. Neuroimage 50, 81-98.

Chen, Z., Calhoun, V., 2018. Effect of spatial smoothing on task fMRI ICA and functional connectivity. Front. Neurosci. 12, 15.

Corbetta, M., Miezin, F.M., Shulman, G.L., Petersen, S.E., 1993. A PET study of visuospatial attention. J. Neurosci. 13, 1202-1226.

Critchley, H.D., Mathias, C.J., Josephs, O., O'Doherty, J., Zanini, S., Dewar, B.K., et al., 2003. Human cingulate cortex and autonomic control: converging neuroimaging and clinical evidence. Brain 126, 2139-2152.

Davidson, R.J., 2004. Well-being and affective style: neural substrates and biobehavioural correlates. Philos. Trans. R. Soc. Lond. B Biol. Sci. 359, 1395-1411.

De Bellis, M.D., 2005. The psychobiology of neglect. Child Maltreat. 10, 150-172.

de Reus, M.A., van den Heuvel, M.P., 2013. The parcellation-based connectome: limitations and extensions. Neuroimage 80,397-404.

Desikan, R.S., Ségonne, F., Fischl, B., Quinn, B.T., Dickerson, B.C., Blacker, D., et al., 2006. An automated labeling system for subdividing the human cerebral cortex on MRI scans into gyral based regions of interest. Neuroimage 31, 968-980.

Dolcos, F., LaBar, K.S., Cabeza, R., 2004. Interaction between the amygdala and the medial temporal lobe memory system predicts better memory for emotional events. Neuron 42, 855-863.

Etkin, A., Prater, K.E., Hoeft, F., Menon, V., Schatzberg, A.F., 2010. Failure of anterior cingulate activation and connectivity with the amygdala during implicit regulation of emotional processing in generalized anxiety disorder. Am. J. Psychiatry 167, 545-554.

Etkin, A., Prater, K.E., Schatzberg, A.F., Menon, V., Greicius, M.D., 2009. Disrupted amygdalar subregion functional connectivity and evidence of a compensatory network in generalized anxiety disorder. Arch. Gen. Psychiatry 66, 1361-1372.

Fornito, A., Zalesky, A., Breakspear, M., 2015. The connectomics of brain disorders. Nat. Rev. Neurosci. 16, 159-172.

Fornito, A., Zalesky, A., Bullmore, E.T., 2010. Network scaling effects in graph analytic studies of human resting-state FMRI data. Front. Syst. Neurosci. 4, 22

Fournier, J.C., Chase, H.W., Almeida, J., Phillips, M.L., 2014. Model specification and the reliability of fmri results: implications for longitudinal neuroimaging studies in psychiatry. PLoS One 9, e105169.

Gao, Q., Xu, Q., Duan, X., Liao, W., Ding, J., Zhang, Z., et al., 2013. Extraversion and neuroticism relate to topological properties of resting-state brain networks. Front. Hum. Neurosci. 7, 257.

Grayson, D.S., Fair, D.A., 2017. Development of large-scale functional networks from birth to adulthood: a guide to the neuroimaging literature. Neuroimage 160, 15-31.

Gong, Q., He, Y., 2015. Depression, neuroimaging and connectomics: a selective overview. Biol. Psychiatry 77, 223-235.

Gottfried, J.A., Dolan, R.J., 2004. Human orbitofrontal cortex mediates extinction learning while accessing conditioned representations of value. Nat. Neurosci. 7, 1144-1152.

Hoffman, D.L., Dukes, E.M., Wittchen, H.U., 2008. Human and economic burden of generalized anxiety disorder. Depress. Anxiety 25, 72-90.

Kambeitz, J., Kambeitz-Ilankovic, L., Cabral, C., Dwyer, D.B., Calhoun, V.D., van den Heuvel, M.P., et al., 2016. Aberrant functional whole-brain network architecture in patients with schizophrenia: a meta-analysis. Schizophr. Bull 42, S13-S21.

Kolaczyk, E.D., Krivitsky, P.N., 2015. On the question of effective sample size in network modeling: an asymptotic inquiry. Stat. Sci. 30, 184-198.

Lei, D., Li, K., Li, L., Chen, F., Huang, X., Lui, S., et al., 2015. Disrupted functional brain connectome in patients with posttraumatic stress disorder. Radiology 276, 818-827.

Li, W., Cui, H., Zhu, Z., Kong, L., Guo, Q., Zhu, Y., et al., 2016. Aberrant functional connectivity between the amygdala and the temporal pole in drug-free generalized anxiety disorder. Front. Hum. Neurosci. 10, 549.

Liu, P., Calhoun, V., Chen, Z., 2017. Functional overestimation due to spatial smoothing of fMRI data. J. Neurosci. Methods 291, 1-12.

Lord, L.D., Allen, P., Expert, P., Howes, O, Broome, M., Lambiotte, R., et al., 2012.
Functional brain networks before the onset of psychosis: a prospective fMRI study with graph theoretical analysis. Neuroimage Clin. 1, 91-98.

Luo, Q., Deng, Z., Qin, J., Wei, D., Cun, L., Qiu, J., et al., 2015. Frequency dependant topological alterations of intrinsic functional connectome in major depressive disorder. Sci. Rep. 5, 9710.

Maheu, F.S., Dozier, M., Guyer, A.E., Mandell, D., Peloso, E., Poeth, K., et al., 2010. A preliminary study of medial temporal lobe function in youths with a history of caregiver deprivation and emotional neglect. Cogn. Affect. Behav. Neurosci. 10, 34-49.

Makovac, E., Meeten, F., Watson, D.R., Herman, A., Garfinkel, S.N.D., Critchley, H.D., et al., 2016a. Alterations in amygdala-prefrontal functional connectivity account for excessive worry and autonomic dysregulation in generalized anxiety disorder. Biol. Psychiatry 80, 786-795.

Makovac, E., Smallwood, J., Watson, D.R., Meeten, F., Critchley, H.D., Ottaviani, C, 2018. The verbal nature of worry in generalized anxiety: insights from the brain. NeuroImage Clin. 17, 882-892.

Makovac, E., Watson, D.R., Meeten, F., Garfinkel, S.N., Cercignani, M., Critchley, H.D., et al., 2016b. Amygdala functional connectivity as a longitudinal biomarker of symptom changes in generalized anxiety. Soc. Cogn. Affect. Neurosci. 11, $1719-1728$.

McClure, E.B., Adler, A., Monk, C.S., Cameron, J., Smith, S., Nelson, E.E., et al., 2007. fMRI Predictors of treatment outcome in pediatric anxiety disorders. Psychopharmacology 191, 97-105.

Meyer, T.J., Miller, M.L., Metzger, R.L., Borkovec, T.D., 1990. Development and validation of the Penn State Worry Questionnaire. Behav. Res. Ther. 28, 487-495.

Menon, V., 2011. Large-scale brain networks and psychopathology: a unifying triple network model. Trends Cogn. Sci. 15, 483-506.

Milad, M.R., Rauch, S.L., 2007. The role of the orbitofrontal cortex in anxiety disorders. Ann. N Y Acad. Sci. 1121, 546-561.

Mochcovitch, M.D., da Rocha Freire, R.C., Garcia, R.F., Nardi, A.E., 2014. A systematic review of fMRI studies in generalized anxiety disorder: evaluating its neural and cognitive basis. J. Affect. Disord. 167, 336-342.

Monk, C.S., Nelson, E.E., McClure, E.B., Mogg, K., Bradley, B.P., Leibenluft, E., et al., 2006. Ventrolateral prefrontal cortex activation and attentional bias in response to angry faces in adolescents with generalized anxiety disorder. Am. J. Psychiatry 163, 1091-1097.

Monk, C.S., Telzer, E.H., Mogg, K., Bradley, B.P., Mai, X., Louro, H.M., Chen, G., McClureTone, E.B., Ernst, M., Pine, D.S., 2008. Amygdala and ventrolateral prefrontal cortex activation to masked angry faces in children and adolescents with generalized anxiety disorder. Arch. Gen. Psychiatry 65, 568-576.

Nitschke, J.B., Sarinopoulos, I., Oathes, D.J., Johnstone, T., Whalen, P.J., Davidson, R.J., Kalin, N.H., 2009. Anticipatory activation in the amygdala and anterior cingulate in generalized anxiety disorder and prediction of treatment response. Am. J. Psychiatry $166,302-310$

Nomura, M., Ohira, H., Haneda, K., Iidaka, T., Sadato, N., Okada, T., et al., 2004 Functional association of the amygdala and ventral prefrontal cortex during cognitive evaluation of facial expressions primed by masked angry faces: an event-related fMR study. NeuroImage 21, 352-363.

Ottaviani, C., Watson, D.R., Meeten, F., Makovac, E., Garfinkel, S.N., Critchley, H.D., 2016. Neurobiological substrates of cognitive rigidity and autonomic inflexibility in generalized anxiety disorder. Biol. Psychol. 119, 31-41.

Palm, M.E., Elliott, R., McKie, S., Deakin, J.F., Anderson, I.M., 2011. Attenuated response to emotional expressions in women with generalized anxiety disorder. Psychol. Med. 41, 1009-1018.

Patriat, R., Molloy, E.K., Meier, T.B., Kirk, G.R., Nair, V.A., Meyerand, M.E., et al., 2013. The effect of resting condition on resting-state fMRI reliability and consistency: a comparison between resting with eyes open, closed, and fixated. Neuroimage 78, 463-473.

Paulus, M.P., Stein, M.B., 2006. An insular view of anxiety. Biol. Psychiatry 60, 383-387.

Peterson, A., Thome, J., Frewen, P., Lanius, R.A., 2014. Resting-state neuroimaging studies: a new way of identifying differences and similarities among the anxiety disorders? Can. J. Psychiatry 59, 294-300.

Pruim, R.H., Mennes, M., Buitelaar, J.K., Beckmann, C.F., 2015. Evaluation of ICA-AROMA and alternative strategies for motion artifact removal in resting state fMRI. Neuroimage 112, 278-287.

Rubinov, M., Sporns, O., 2010. Complex network measures of brain connectivity: uses and interpretations. Neuroimage 52, 1059-1069.

Segarra, S., Ribeiro, A., 2016. Stability and continuity of centrality measures in weighted graphs. IEEE Trans. Sig. Process 64, 543-555.

Servaas, M.N., Riese, H., Renken, R.J., Wichers, M., Bastiaansen, J.A., Figueroa, C.A., et al., 2017. Associations between daily affective instability and connectomics in functional subnetworks in remitted patients with recurrent major depressive disorder. Neuropsychopharmacology 42, 2583-2592.

Smiths, S.M., Nichols, T.E., 2009. Threshold-free cluster enhancement: addressing problems of smoothing, threshold dependence and localisation in cluster inference. Neuroimage 44, 83-98.

Spielberg, J.M., Beall, E.B., Hulvershorn, L.A., Altinay, M., Karne, H., Anand, A., 2016. Resting state brain network disturbances related to hypomania and depression in medication-free bipolar disorder. Neuropsychopharmacology 41, 3016-3024.

Spielberger, C.D., 1983. State-Trait Anxiety Inventory. A Comprehensive Bibliography. Consulting Psychologists Press, Palo Alto.

Sporns, O., Honey, C.J., Kötter, R., 2007. Identification and classification of hubs in brain networks. PLoS One 2, e1049.

Steiger, J.H., 1980. Tests for comparing elements of a correlation matrix. Psychol. Bull. 87, 245-251.

Sylvester, C.M., Corbetta, M., Raichle, M.E., Rodebaugh, T.L., Schlaggar, B.L., Sheline, 
Y.I., et al., 2012. Functional network dysfunction in anxiety and anxiety disorders. Trends Neurosci. 35, 527-535.

van Beljouw, I.M., Verhaak, P.F., Cuijpers, P., van Marwijk, H.W., Penninx, B.W., 2010 The course of untreated anxiety and depression, and determinants of poor one-year outcome: a one-year cohort study. BMC Psychiatry 10, 86.

van den Heuvel, M.P., de Lange, S.C., Zalesky, A., Seguin, C., Yeo, B.T.T., Schmidt, R., 2017. Proportional thresholding in resting-state fMRI functional connectivity networks and consequences for patient-control connectome studies: issues and recommendations. Neuroimage 152, 437-449.

Via, E., Fullana, M.A., Goldberg, X., Tinoco-González, D., Martínez-Zalacaín, I., SorianoMas, C., et al., 2018. Ventromedial prefrontal cortex activity and pathological worry in generalised anxiety disorder. Br. J. Psychiatry 213, 437-443.

Wang, Z., Suh, J., Li, Z., Li, Y., Franklin, T., O'Brien, C., et al., 2015. A Hyper-connected but less efficient small-world network in the substance-dependent brain. Drug Alcohol Depend. 152, 102-108.

Watts, D.J., Strogatz, S.H., 1998. Collective dynamics of "small-world" networks. Nature 393, 440-442.

Wolpert, D.M., Goodbody, S.J., Husain, M., 1998. Maintaining internal representations: the role of the human superior parietal lobe. Nat. Neurosci. 1, 529-533.

Xing, M., Tadayonnejad, R., MacNamara, A., Ajilore, O., DiGangi, J., Phan, K.L., et al., 2016. Resting-state theta band connectivity and graph analysis in generalized social anxiety disorder. Neuroimage Clin. 13, 24-32.

Yang, X., Liu, J., Meng, Y., Xia, M., Cui, Z., Wu, X., et al., 2017. Network analysis reveals disrupted functional brain circuitry in drug-naive social anxiety disorder. Neuroimage Advance online publication. https://doi.org/10.1016/j.neuroimage. 2017.12.011.

Yang, Y., Zhang, X., Zhu, Y., Dai, Y., Liu, T., Wang, Y., 2015. Cognitive impairment in generalized anxiety disorder revealed by event-related potential N270. Neuropsychiatr. Dis. Treat. 11, 1405-1411.

Yao, Z., Liao, M., Hu, T., Zhang, Z., Zhao, Y., Zheng, F., et al., 2017. An effective method to identify adolescent generalized anxiety disorder by temporal features of dynamic functional connectivity. Front. Hum. Neurosci. 11, 492.

Yun, J.Y., Kim, J.C., Ku, J., Shin, J.E., Kim, J.J., Choi, S.H., 2017. The left middle temporal gyrus in the middle of an impaired social-affective communication network in social anxiety disorder. J. Affect. Disord. 214, 53-59.

Zalesky, A., Fornito, A., Bullmore, E.T., 2010. Network-based statistic: identifying differences in brain networks. Neuroimage 53, 1197-1207.

Zhu, H., Qiu, C., Meng, Y., Yuan, M., Zhang, Y., Ren, Z., et al., 2017. Altered topological properties of brain networks in social anxiety disorder: a resting-state functional MRI study. Sci. Rep. 7, 43089.

Zhu, J., Wang, C., Liu, F., Qin, W., Li, J., Zhuo, C., 2016. Alterations of functional and structural networks in schizophrenia patients with auditory verbal hallucinations. Front. Hum. Neurosci. 10, 114.

Zuo, X.N., Xing, X.X., 2014. Test-retest reliabilities of resting-state FMRI measurements in human brain functional connectomics: a systems neuroscience perspective. Neurosci. Biobehav. Rev. 45, 100-118. 